\title{
Human Codon Usage: The Genetic Basis of Pathogen Latency
}

\author{
Darja Kanduc ${ }^{1}$ \\ ${ }^{1}$ Department of Biosciences, Biotechnologies and Biopharmaceutics, \\ University of Bari, Bari, Italy
}

Global Med Genet 2021;8:109-115.
Address for correspondence Darja Kanduc, PhD, Department of Biosciences, Biotechnologies and Biopharmaceutics, University of Bari, Via Orabona 4, Bari 70125, Italy (e-mail: dkanduc@gmail.com).

\begin{abstract}
Keywords

- pathogen latency

- (re)activation

- protein synthesis

- cross-reactivity

- codon usage

- tRNAs

- codon optimization
\end{abstract}

Infectious diseases pose two main compelling issues. First, the identification of the molecular factors that allow chronic infections, that is, the often completely asymptomatic coexistence of infectious agents with the human host. Second, the definition of the mechanisms that allow the switch from pathogen dormancy to pathologic (re) activation. Furthering previous studies, the present study (1) analyzed the frequency of occurrence of synonymous codons in coding DNA, that is, codon usage, as a genetic tool that rules protein expression; (2) described how human codon usage can inhibit protein expression of infectious agents during latency, so that pathogen genes the codon usage of which does not conform to the human codon usage cannot be translated; and (3) framed human codon usage among the front-line instruments of the innate immunity against infections. In parallel, it was shown that, while genetics can account for the molecular basis of pathogen latency, the changes of the quantitative relationship between codon frequencies and isoaccepting tRNAs during cell proliferation offer a biochemical mechanism that explains the pathogen switching to (re)activation. Immunologically, this study warns that using codon optimization methodologies can (re)activate, potentiate, and immortalize otherwise quiescent, asymptomatic pathogens, thus leading to uncontrollable pandemics.

\section{Introduction}

Infectious pathogens-from viruses to fungi-establish latent infections in the human host and can, then, reactivate with severe pathologic sequelae. To quote some examples:

- Herpes simplex virus (HSV) types 1 and 2 that are capable of establishing lifelong infection primarily in neurons, and (re)activation of which may be accompanied by herpes encephalitis and recurrent vesicular eruptions in the orolabial and genital mucosa. ${ }^{1-3}$

- Likewise, human herpesvirus 6A and 6B establish latency in the central nervous system, with potential to reactivate and cause multiple sclerosis and epilepsy, respectively. ${ }^{4}$
- Human cytomegalovirus (HCMV) is a nearly ubiquitous $\beta$ herpesvirus capable of establishing a latent phase in humans. ${ }^{5,6}$ HCMV (re)activation may associate with both systemic and end-organ severe diseases. ${ }^{7-9}$

- Epstein-Barr's virus establishes and maintains latency in B cells, and its (re)activation may associate with several malignant tumors ${ }^{10,11}$ and a vast number of pathologies. ${ }^{12,13}$

- Mycobacterium tuberculosis is able to persist for the lifetime of the host, indicating that this pathogen has substantial molecular mechanisms to resist host-inflicted damage. Infection of humans with $M$. tuberculosis is frequent and can also lead to brain tuberculomas and meningitis. ${ }^{14}$
DOI https://doi.org/

10.1055/s-0041-1729753.

ISSN 2699-9404. (c) 2021. The Author(s).

This is an open access article published by Thieme under the terms of the Creative Commons Attribution License, permitting unrestricted use, distribution, and reproduction so long as the original work is properly cited. (https://creativecommons.org/licenses/by/4.0/)

Georg Thieme Verlag KG, Rüdigerstraße 14, 70469 Stuttgart, Germany 
- Toxoplasma gondii can remain dormant for years as bradyzoite within the host. ${ }^{15}$ Toxoplasma gondii (re)activation may associate with chorioretinitis, encephalitis, and neuropsychiatric disorders such as schizophrenia. ${ }^{16}$

- Plasmodium falciparum can reactivate during pregnancy after years of latency. ${ }^{17}$

- Cryptococcus neoformans is a common central nervous system pathogen and causes fatal fungal meningoencephalitis, especially in immunocompromised subjects. ${ }^{18,19}$

Pathologically, the disease burden related to pathogen (re) activation is overwhelming and eradication of chronic latent infections is a health top priority, especially when considering that latent infections are widespread in all over the world. ${ }^{20-25}$ In general, persistent pathogen infections have been associated with an immune response that is unable to react with pathogen-infected cells. ${ }^{26}$ In the years, escape from immune surveillance has been explained as possibly due to inhibition of host cell human leukocyte antigen class II expression $^{27}$; suppression of the expression of multiple genes that are important for antigen processing and presentation $^{28}$; selective elimination of Th-cells by apoptosis ${ }^{29}$; escape from cytotoxic $\mathrm{T}$ lymphocytes ${ }^{30}$; antigenic drift $^{31}$; production of immunosuppressive molecules ${ }^{32,33}$; targeting of dendritic cell-specific intercellular-adhesion-molecule-3grabbing nonintegrin ${ }^{34}$; and hijacking of the lipoxygenase machinery of the host, ${ }^{35}$ inter alia. On the whole, this corpus of data contributed important knowledge advancement of virology and microbiology, but unfortunately, the mechanism(s) underlying pathogen quiescence remain elusive. ${ }^{36}$

Metabolically, it has been repeatedly observed that the pathogen persistence in the human host is characterized by restriction of pathogen protein production ${ }^{37}$ so that passage from latency to (re)activation requires ex novo protein synthesis. ${ }^{38,39}$ Then, it is assumed that chronic latent infections cannot be eradicated since, given the minimal expression of pathogen proteins, the host immune system cannot recognize the infected cell through the pathogen peptides presented on its cell surface. That is, antigenemia and patient immune responses are correlated, ${ }^{40,41}$ by being the outcome of the antibody response: a question of antigen dose. ${ }^{42}$ Simply put, in absentia of pathogen protein synthesis, there is no pathogen target that might evoke antipathogen attacks by the host immune system and, as a consequence, latent infections cannot be eradicated.

In contrast with this view and based on reports $7,8,12,13$ documenting a high level of peptide sharing between pathogens and human proteins, the author's laboratory studies on cytomegalovirus (CMV) $)^{43,44}$ analyzed the restriction of CMV protein synthesis as a device imposed via human codon usage purposely to block immune responses, with the ultimate aim of protecting the host from potential harmful autoimmune cross-reactions. ${ }^{45-48}$ Indeed, lack of pathogen protein expression would prevent not only immune attacks against the pathogen proteins but would also inhibit crossreactive autoimmune reactions against the host proteins sharing sequences with the pathogens. Expanding these studies, here the human codon usage has been compared with that of four genes coding for (re)activation-related proteins from HSV-1, M. tuberculosis, P. falciparum, and C. neoformans, respectively. Results document and confirm the role of the human codon usage in determining the silencing of pathogen protein expression, and highlight the correlation between codon frequencies and amounts of the corresponding isoaccepting tRNA as the biochemical mechanism that can trigger pathogen (re)activation.

\section{Methods}

The gene coding sequences (open reading frames, ORFs) from the following four pathogen proteins were analyzed for codon usage:

- major viral transcription factor ICP4 (ICP4; UniProt: P08392, ICP4_HHV11, GenBank: AAA96675.1) from HSV-1 (NCBI:txid10298);

- transcriptional regulator WhiB5 (WhiB5; UniProt: P71592; WHB5A_MYCTU; GenBank: CCP42744.1) from M. tuberculosis (NCBI: txid83332);

- proliferation-associated protein 2 g4(2 g4; UniProt: Q8ILI2_PLAF7; NCBI reference sequence: XM_001348399.1) from $P$. falciparum (NCBI:txid36329);

- eukaryotic translation initiation factor 3 subunit A (eIF3a; UniProt: POCN42, EIF3A_CRYNJ; NCBI reference sequence: XM_570890.1) from C. neoformans (NCBI:txid214684).

The ORF of the human protein Sushi repeat-containing protein SRPX2 (SRPX2; UniProt: 060687; SRPX2_HUMAN; NCBI Reference Sequence: NM_014467.3) was analyzed as a control.

Codon usage of the Homo sapiens ORFeome was obtained from the international DNA sequence database (http://www. kazusa.or.jp/codon/). ${ }^{49}$ Codon usage of the ORFs coding for the above-listed proteins was obtained using GeneInfinity program (http://www.geneinfinity.org). Protein details were obtained from UniProt resource (www.uniprot.org/). ${ }^{50}$

\section{Results}

\section{The Genetic Basis of Pathogen Latency}

Four ORFs coding for proteins that are representative of viral, bacterial, protozoan, and fungal pathogens, respectively, were analyzed for codon usage. Results were compared with the codon usage of the human ORFeome. The four pathogen proteins were selected because of their crucial role in pathogen (re)activation, that is, specifically:

- HSV-1 ICP4 is a major viral transcription factor that is necessary for the transition from immediate early gene transcription to later viral gene transcription; ${ }^{51}$

- WhiB5 is a transcriptional regulator that contributes to $M$. tuberculosis virulence and (re)activation; ${ }^{52}$

- $2 \mathrm{~g} 4$ is a proliferation-associated protein that belongs to the proteases implicated in the $P$. falciparum erythrocytic replication cycle including merozoite egress from schizonts, host cell invasion by merozoites, and hemoglobin degradation; ${ }^{53,54}$ 
- C. neoformans eIF3a is a subunit of the eukaryotic translation initiation factor 3 (eIF-3) complex. The eIF-3 complex specifically targets a subset of mRNAs involved in the cell proliferation. ${ }^{55}$

In addition, the human protein SRPX2 was used as a control because it is expressed in the brain, an organ where pathogen quiescence preferentially occurs. In particular, SRPX2 is expressed in neurons of the rolandic area of the brain with a role in the perisylvian region, critical for language and cognitive development. ${ }^{56}$

The comparative pathogen versus human codon usage pattern is illustrated in -Fig. $\mathbf{1}$ and numerically tabulated in - Supplementary Table S1 (online only).

-Fig. 1 shows four fundamental points:

- All of the 61 codons that specify the 20 amino acids in the genetic code are used in the human ORFeome (-Fig. 1, panel 1).
- The control, that is, the neuronal human SRPX2 ORF, conforms to the human ORFeome in the codon choices (-Fig. 1, panel 2).

- In contrast, the four proteins derived from pathogens and essential for (re)activation ${ }^{51-55}$ are coded by ORFs characterized by codon usage patterns markedly different from those of the human ORFeome and neuronal human SRPX2 ORF, with many codons being unused and a few codons being overused (-Fig. 1, panels 3-6 vs. panels 1 and 2).

- The codon usages of the four ORFs coding for the pathogen proteins are strikingly different among themselves and have no codon choices in common and, rather, each of the four ORFs uses a highly specific codon pattern (-Fig. 1, panels 3-6).

In sum, - Fig. 1 shows that the usage of synonymous codons in ORFs that code for (re)activation-related pathogen proteins differs from the human codon usage. A striking

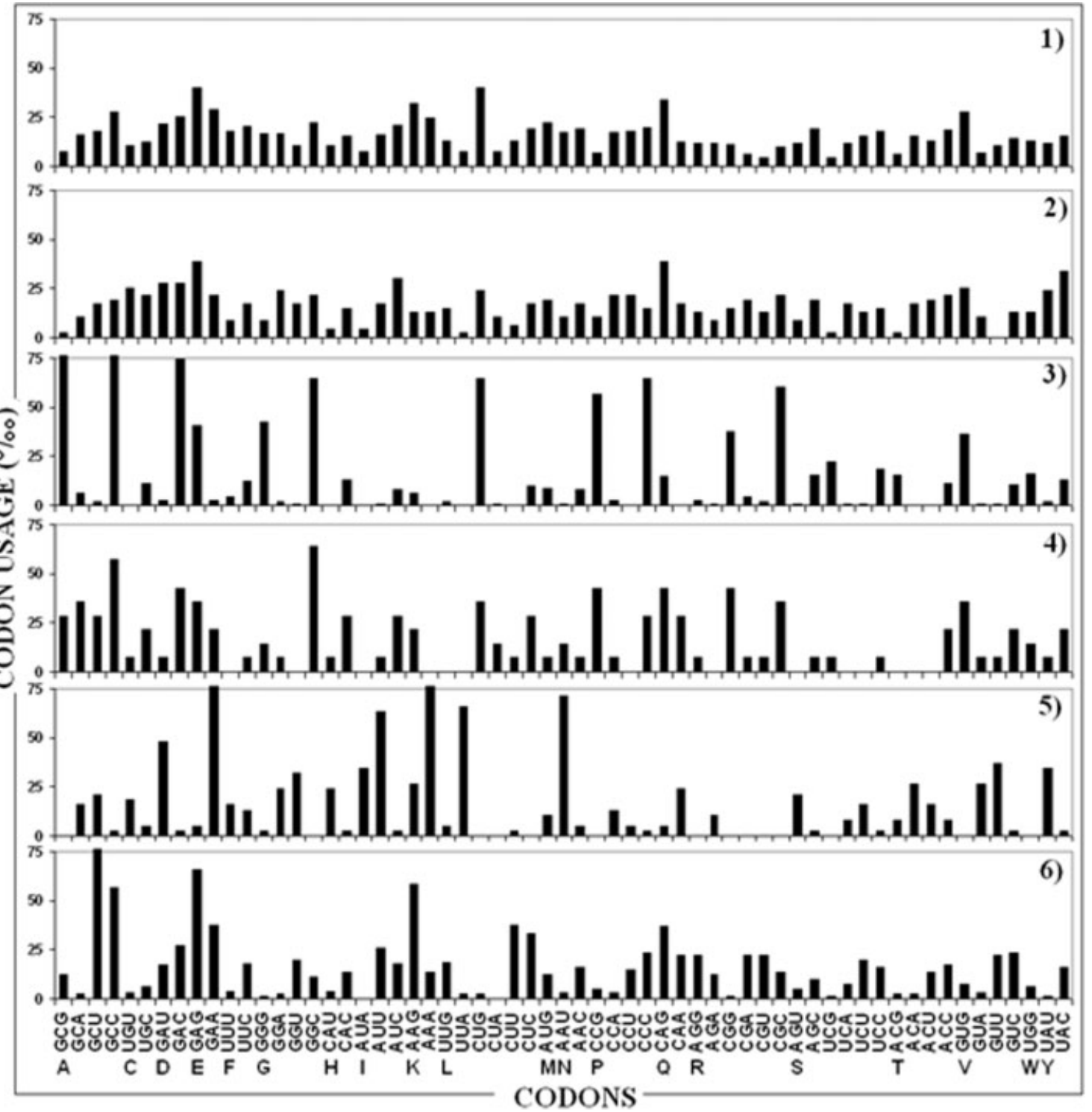

Fig. 1 Codon usage of (1) human ORFeome, and ORF coding for: (2) human SRPX2; (3) HSV-1 ICP4; (4) M. tuberculosis WhiB5; (5) P. falciparum 2 g4; and (6) C. neoformans elF3a. Codon usage is expressed as codon frequency per thousand. In the abscissa, amino acids given in one-letter code. 
example is the HSV-1 ICP4 ORF that preferentially uses the Ala codon GCG (76.98\%) that, instead, is rarely used in the human ORFeome as well as in the human neuronal SRPX2 ORF (7.37 and 2.15\%o, respectively) (- Fig. 1, panel 3 vs. panels 1 and 2, and - Supplementary Table S1). Likewise, deviations from the human codon usage are evident in the bacterial, protozoan, and fungal ORFs coding for the (re) activation-related proteins analyzed here (-Fig. 1, panels 4-6 vs. panels 1 and 2, and -Supplementary Table S1).

Then, given the long-standing notion that codon usage is a basic determinant of gene expression, ${ }^{57-62}$ results illustrated in -Fig. 1 and tabulated in - Supplementary Table $\mathbf{S 1}$ provide physical reality to the working hypothesis according to which deviation from the host usage of synonymous codons represents a powerful genetic constraint capable of blocking pathogen protein synthesis in the human host. In fact, as a documented known rule, ${ }^{63}$ the ORFs/ORFeome of each biological entity-from proteins to proteomes, from viruses to humans-are characterized by specific sets of synonymous codons that determine/inhibit/modulate the protein expression pattern in cells, tissues, and organisms. Accordingly, ORFs that preferentially use optimal synonymous codons (i.e., the most abundant ones) are easily expressed, while ORFs that do not match with the host ORFeome and use nonoptimal synonymous codons (i.e., the rare ones) will be expressed at a very limited extent, if any.

\section{Biochemical Basis of Pathogen (Re)Activation}

Data illustrated in -Fig. 1 and - Supplementary Table S1 locate the molecular basis of the lack of pathogen protein expression in the human host as due to different codon usages, but by themselves do not explain how pathogen usage of suboptimal codons can lead to a block of pathogen protein synthesis.

Actually, since $1980 \mathrm{~s},{ }^{64-68}$ it was demonstrated that, mechanistically, the basis for the correlation between rarely used codons and restricted protein expression (or, vice versa, highly used codons and high protein expression) resides in the quantitative matching between synonymous codons and isoaccepting tRNAs. That is, codon frequencies correlate with the amounts of the corresponding isoaccepting tRNA so that optimal, highly used codons correlate with abundant isoaccepting tRNAs, whereas rarely used, lowfrequency codons correlate with low amounts of the corresponding isoaccepting tRNAs. Such a quantitative relationship between codons and isoaccepting tRNAs implies that the composition of the tRNA isoacceptor pools has to change in order pathogen protein expression can be resumed. In effect, changes in the composition of the tRNA isoacceptor pools occur under growth conditions. As documented in - Fig. 2, changes of tRNA isoaccepting species, as both relative percentage of total tRNA and absolute concentration, occur during cell proliferation induced by partial hepatectomy. ${ }^{69}$

Specifically, - Fig. 2 shows that tRNAs which are abundant under quiescence decrease during cell proliferation, and vice versa, so that cell proliferation provides a metabolic window for resumption of pathogen protein expression.
In this experimentally validated perspective, it assumes a crucial importance the fact that pathogen (re)activation is mostly associated with immunosuppressive treatments, ${ }^{70-77}$ that is, with treatments that in general implicate administration of glucocorticoids. As a matter of fact, it is well known that glucocorticosteroids can induce cell proliferation ${ }^{78-91}$ so that, consequently, it can induce proliferation-associated tRNA changes and favor pathogen protein expression and (re)activation. Therefore, in a clinical context, the present study might also help understand the pathogen (re)activation phenomenon in infected fetuses and newborns (i.e., in organisms growing rapidly) $)^{7}$ and pregnancy, ${ }^{17}$ as well as in subjects treated with glucocorticoids following, for example, transplant procedures. $^{75,77}$

\section{Conclusion}

A leitmotiv of the research conducted in the author's laboratory since $2000^{92}$ is that, following immune responses against infectious pathogens, the extremely high level of peptide sharing between human proteins and infectious agents ${ }^{92-94}$ can cause harmful autoimmune cross-reactions and severe pathologies in the human host. ${ }^{7,8,12,13,48}$ In this scientific context and using CMV as a research model, ${ }^{43,44}$ data have been obtained in favor of the hypothesis that, to avoid cross-reactivity, expression of genes essential for viral (re)activation is purposely blocked because of a viral usage of synonymous codons different from that of the host. Here, the present study provides further evidences in favor of such working hypothesis by comparatively analyzing the human codon usage to that of four ORFs coding for (re)activationrelated proteins derived, respectively, from HSV-1, M. tuberculosis, P. falciparum, and C. neoformans. Indeed, the data illustrated in - Fig. 1 substantiate the concept that human codon usage is a main factor able to block pathogen protein expression in the human host, in this way avoiding potential immune response-associated cross-reactions and, consequently, allowing a pacific, unharmful, quiet coexistence between potentially dangerous pathogens and the human host.

In practice, pathogen-restricted protein synthesis emerges as a crucial protective phenomenon that avoids immune responses and the associated potential autoimmunity. Accordingly, resumption of pathogen protein synthesis by the fine tuning of the quantitative relationship between codons and isoaccepting tRNAs via cell proliferation (-Fig. 2), that is, under proliferative conditions determined by therapeutical treatments (such as glucocorticoids) or physiological cell proliferation (growth, pregnancy) may trigger pathogen virulence by evoking antipathogen immune responses able to cross-react with the host proteins.

So, as a logical conclusion, the genetic basis that specifically characterizes the human gene expression, that is, the human codon usage, has the value of a powerful first-line defense in the human innate immunity. Useless to say, clinically, the present study and conclusions invite to revise approaches currently used for managing infectious diseases and related pathologies. This is all the more so in light of the 


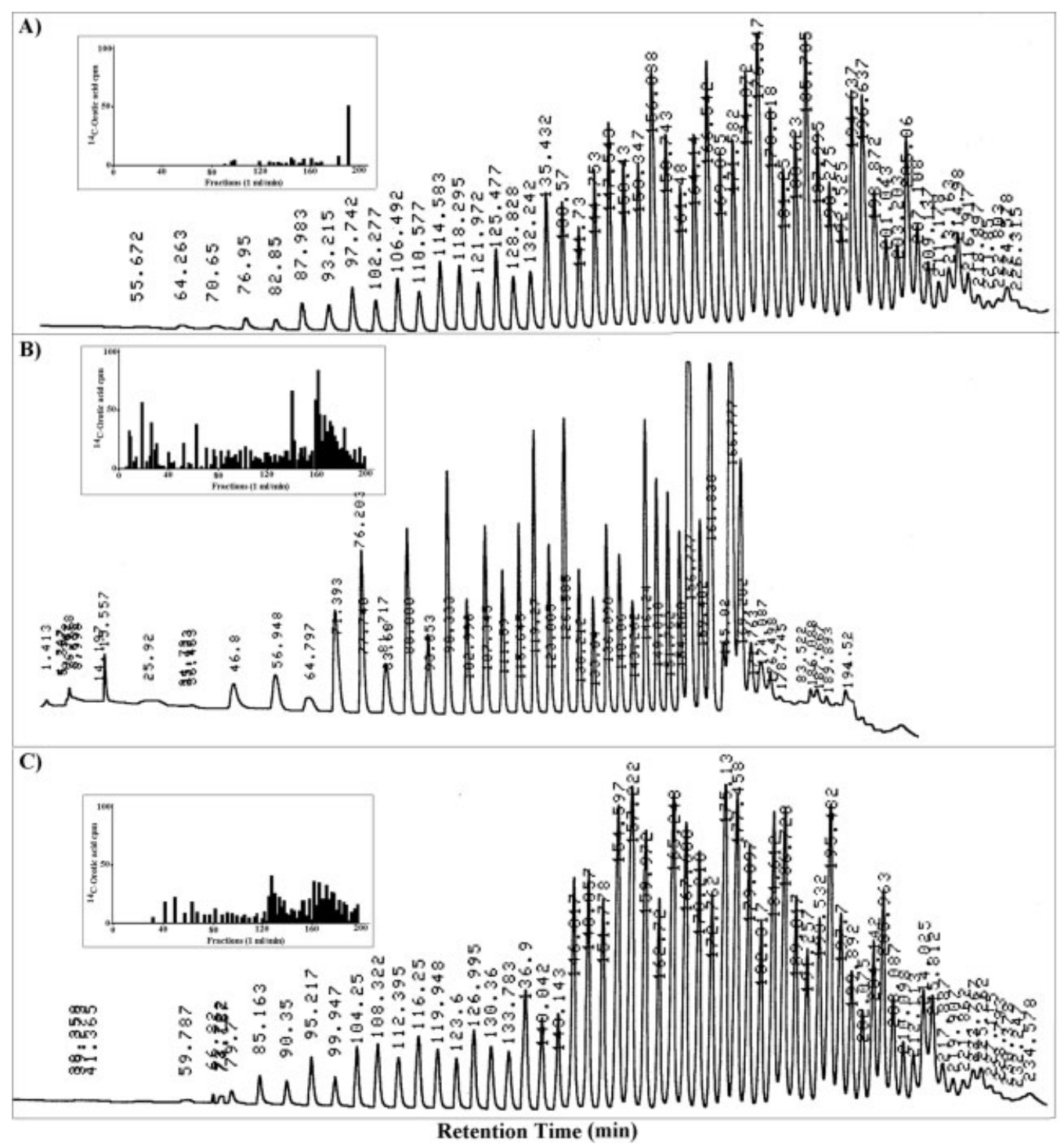

Fig. 2 Changes of tRNA species during cell proliferation. High-performance liquid chromatography of (A) hepatic cytoplasmic tRNA population from quiescent nontreated, and from proliferating liver at (B) 20 hours and (C) 1 week following partial hepatectomy. In each panel, the inset reports $\left[{ }^{14} \mathrm{C}\right]$ orotic acid incorporation into tRNA. (From Kanduc, ${ }^{69}$ with experimental details in the reference.)

current (re)emerging infectious threats such as the severe acute respiratory syndrome-related coronavirus 2. ${ }^{95,96}$

\section{Funding \\ None.}

Conflict of Interest

None declared.

\section{References}

1 Cohen JI. Herpesvirus latency. J Clin Invest 2020;130(07): 3361-3369

2 Maroui MA, Callé A, Cohen C, et al. Latency entry of herpes simplex virus 1 is determined by the interaction of its genome with the nuclear environment. PLoS Pathog 2016;12(09):e1005834

3 Dogrammatzis C, Saleh S, Deighan C, Kalamvoki M. Diverse populations of extracellular vesicles with opposite functions during herpes simplex virus 1 infection. J Virol 2021;95(06):x
4 Dunn N, Kharlamova N, Fogdell-Hahn A. The role of herpesvirus 6A and 6B in multiple sclerosis and epilepsy. Scand J Immunol 2020;92(06):e12984

5 Elder E, Sinclair J. HCMV latency: what regulates the regulators? Med Microbiol Immunol (Berl) 2019;208(3-4):431-438

6 Semmes EC, Hurst JH, Walsh KM, Permar SR. Cytomegalovirus as an immunomodulator across the lifespan. Curr Opin Virol 2020; 44:112-120

7 Lucchese G, Kanduc D. Cytomegalovirus infection: the neurodevelopmental peptide signatures. Curr Drug Discov Technol 2018;15(03):251-262

8 Kanduc D. From anti-SARS-CoV-2 immune responses to COVID19 via molecular mimicry. Antibodies (Basel) 2020;9(03):33

9 Gugliesi F, Coscia A, Griffante G, et al. Where do we stand after decades of studying human cytomegalovirus? Microorganisms 2020;8(05):685

10 Di Pietro A. Epstein-Barr virus promotes B cell lymphomas by manipulating the host epigenetic machinery. Cancers (Basel) 2020;12(10):3037

11 Rezk SA, Weiss LM. EBV-associated lymphoproliferative disorders: update in classification. Surg Pathol Clin 2019;12(03):745-770 
12 Kanduc D. From hepatitis C virus immunoproteomics to rheumatology via cross-reactivity in one table. Curr Opin Rheumatol 2019;31(05):488-492

13 Kanduc D. Proteome-wide Epstein-Barr virus analysis of peptide sharing with human systemic lupus erythematosus autoantigens. Isr Med Assoc J 2019;21(07):444-448

14 Vasconcelos G, Santos L, Couto C, Cruz M, Castro A. Miliary brain tuberculomas and meningitis: tuberculosis beyond the lungs. Eur J Case Rep Intern Med 2020;7(12):001931

15 Jeffers V, Tampaki Z, Kim K, Sullivan WJ Jr. A latent ability to persist: differentiation in Toxoplasma gondii. Cell Mol Life Sci 2018;75(13):2355-2373

16 Zhao XY, Ewald SE. The molecular biology and immune control of chronic Toxoplasma gondii infection. J Clin Invest 2020;130(07): 3370-3380

17 Al Hammadi A, Mitchell M, Abraham GM, Wang JP. Recrudescence of Plasmodium falciparum in a primigravida after nearly 3 years of latency. Am J Trop Med Hyg 2017;96(03):642-644

18 Alanio A. Dormancy in Cryptococcus neoformans: 60 years of accumulating evidence. J Clin Invest 2020;130(07):3353-3360

19 Lee KT, Hong J, Lee DG, et al. Fungal kinases and transcription factors regulating brain infection in Cryptococcus neoformans. Nat Commun 2020;11(01):1521

20 Looker KJ, Magaret AS, May MT, et al. Global and regional estimates of prevalent and incident herpes simplex virus type 1 infections in 2012. PLoS One 2015;10(10):e0140765

21 Kuri A, Jacobs BM, Vickaryous N, et al. Epidemiology of EpsteinBarr virus infection and infectious mononucleosis in the United Kingdom. BMC Public Health 2020;20(01):912

22 Shwab EK, Saraf P, Zhu XQ et al. Human impact on the diversity and virulence of the ubiquitous zoonotic parasite Toxoplasma gondii. Proc Natl Acad Sci U S A 2018;115(29):E6956-E6963

23 Pleyer U, Gross U, Schlüter D, Wilking H, Seeber F. Toxoplasmosis in Germany. Dtsch Arztebl Int 2019;116(25):435-444

24 Turdumambetova GK, Osmanov A, Denning DW. The burden of serious fungal infections in Kyrgyzstan. J Fungi (Basel) 2019;5 (03):66

25 Pappas PG. Cryptococcal infections in non-HIV-infected patients. Trans Am Clin Climatol Assoc 2013;124:61-79

26 Oldstone MB. Anatomy of viral persistence. PLoS Pathog 2009;5 (07):e1000523

27 Sedmak DD, Guglielmo AM, Knight DA, Birmingham DJ, Huang EH, Waldman WJ. Cytomegalovirus inhibits major histocompatibility class II expression on infected endothelial cells. Am J Pathol 1994; 144(04):683-692

28 Rotem-Yehudar R, Groettrup M, Soza A, Kloetzel PM, Ehrlich R. LMP-associated proteolytic activities and TAP-dependent peptide transport for class $1 \mathrm{MHC}$ molecules are suppressed in cell lines transformed by the highly oncogenic adenovirus 12. J Exp Med 1996;183(02):499-514

29 Copeland KF, Heeney JL. T helper cell activation and human retroviral pathogenesis. Microbiol Rev 1996;60(04):722-742

30 Oldstone MB. How viruses escape from cytotoxic T lymphocytes: molecular parameters and players. Virology 1997;234(02): 179-185

31 Voeten JT, Bestebroer TM, Nieuwkoop NJ, Fouchier RA, Osterhaus AD, Rimmelzwaan GF. Antigenic drift in the influenza A virus (H3N2) nucleoprotein and escape from recognition by cytotoxic $\mathrm{T}$ lymphocytes. J Virol 2000;74(15):6800-6807

32 Large MK, Kittlesen DJ, Hahn YS. Suppression of host immune response by the core protein of hepatitis $C$ virus: possible implications for hepatitis C virus persistence. J Immunol 1999; 162(02):931-938

33 Means RE, Choi JK, Nakamura H, Chung YH, Ishido S, Jung JU. Immune evasion strategies of Kaposi's sarcoma-associated herpesvirus. Curr Top Microbiol Immunol 2002;269:187-201
34 van Kooyk Y, Appelmelk B, Geijtenbeek TB. A fatal attraction: Mycobacterium tuberculosis and HIV-1 target DC-SIGN to escape immune surveillance. Trends Mol Med 2003;9(04):153-159

35 Aliberti J. Host persistence: exploitation of anti-inflammatory pathways by Toxoplasma gondii. Nat Rev Immunol 2005;5(02): 162-170

36 Singh N, Tscharke DC. Herpes simplex virus latency is noisier the closer we look. J Virol 2020;94(04):e01701-e01719

37 Jiang G, Santos Rocha C, Hirao LA, et al. HIV exploits antiviral host innate GCN2-ATF4 signaling for establishing viral replication early in infection. MBio 2017;8(03):e015-e016

38 Qin Q Penkert RR, Kalejta RF. Heterologous viral promoters incorporated into the human cytomegalovirus genome are silenced during experimental latency. J Virol 2013;87(17): 9886-9894

39 Raja P, Lee JS, Pan D, Pesola JM, Coen DM, Knipe DM. A herpesviral lytic protein regulates the structure of latent viral chromatin. MBio 2016;7(03):e00633-e16

40 Tricou V, Minh NN, Farrar J, Tran HT, Simmons CP. Kinetics of viremia and NS1 antigenemia are shaped by immune status and virus serotype in adults with dengue. PLoS Negl Trop Dis 2011;5 (09):e1309

41 Rodriguez-Osorio M, Gómez-García V, Rojas J, Ramajo-Martin V. Humoral immune response and antigenemia in sheep experimentally infected with Schistosoma bovis. Cross-reactivity with Fasciola hepatica antigens. J Parasitol 1999;85(03):585-587

42 Fehr T, Ochsenbein AF. Outcome of the antibody response: a question of antigen dose and distribution. Trends Immunol 2004;25(04):165-166, author reply 167-168

43 Kanduc D. Role of codon usage and tRNA changes in rat cytomegalovirus latency and (re)activation. J Basic Microbiol 2016;56(06): 617-626

44 Kanduc D. Rare human codons and HCMV translational regulation. J Mol Microbiol Biotechnol 2017;27(04):213-216

45 Regner M. Cross-reactivity in T-cell antigen recognition. Immunol Cell Biol 2001;79(02):91-100

46 Kamradt T, Volkmer-Engert R. Cross-reactivity of T lymphocytes in infection and autoimmunity. Mol Divers 2004;8(03):271-280

47 Vojtek I, Buchy P, Doherty TM, Hoet B. Would immunization be the same without cross-reactivity? Vaccine 2019;37(04):539-549

48 Kanduc D. Peptide cross-reactivity: the original sin of vaccines. Front Biosci (Schol Ed) 2012;4:1393-1401

49 Nakamura Y, Gojobori T, Ikemura T. Codon usage tabulated from international DNA sequence databases: status for the year 2000 . Nucleic Acids Res 2000;28(01):292

50 UniProt Consortium. UniProt: the universal protein knowledgebase in 2021. Nucleic Acids Res 2021;49(D1):D480-D489

51 Wagner LM, Bayer A, Deluca NA. Requirement of the N-terminal activation domain of herpes simplex virus ICP4 for viral gene expression. J Virol 2013;87(02):1010-1018

52 Casonato S, Cervantes Sánchez A, Haruki H, et al. WhiB5, a transcriptional regulator that contributes to Mycobacterium tuberculosis virulence and reactivation. Infect Immun 2012;80(09): 3132-3144

53 Weißbach T, Golzmann A, Bennink S, Pradel G, Julius Ngwa C. Transcript and protein expression analysis of proteases in the blood stages of Plasmodium falciparum. Exp Parasitol 2017;180:33-44

$54 \mathrm{Li} \mathrm{H}$, Child MA, Bogyo M. Proteases as regulators of pathogenesis: examples from the Apicomplexa. Biochim Biophys Acta 2012; 1824(01):177-185

55 Lee AS, Kranzusch PJ, Cate JH. eIF3 targets cell-proliferation messenger RNAs for translational activation or repression. Nature 2015;522(7554):111-114

56 Roll P, Rudolf G, Pereira S, et al. SRPX2 mutations in disorders of language cortex and cognition. Hum Mol Genet 2006;15(07): 1195-1207 
57 Henry I, Sharp PM. Predicting gene expression level from codon usage bias. Mol Biol Evol 2007;24(01):10-12

58 Quax TE, Claassens NJ, Söll D, van der Oost J. Codon bias as a means to fine-tune gene expression. Mol Cell 2015;59(02):149-161

59 Zhou Z, Dang Y, Zhou M, et al. Codon usage is an important determinant of gene expression levels largely through its effects on transcription. Proc Natl Acad Sci U S A 2016;113(41): E6117-E6125

60 Shin YC, Bischof GF, Lauer WA, Desrosiers RC. Importance of codon usage for the temporal regulation of viral gene expression. Proc Natl Acad Sci U S A 2015;112(45):14030-14035

61 Kames J, Alexaki A, Holcomb DD, et al. TissueCoCoPUTs: novel human tissue-specific codon and codon-pair usage tables based on differential tissue gene expression. J Mol Biol 2020;432(11): 3369-3378

62 Liu Y, Yang Q Zhao F. Synonymous but not silent: the codon usage code for gene expression and protein folding. Annu Rev Biochem 2021;90. Doi: 10.1146/annurev-biochem-071320-112701

63 Grantham R, Gautier C, Gouy M, Jacobzone M, Mercier R. Codon catalog usage is a genome strategy modulated for gene expressivity. Nucleic Acids Res 1981;9(01):r43-r74

64 Ikemura T. Correlation between the abundance of yeast transfer RNAs and the occurrence of the respective codons in protein genes. Differences in synonymous codon choice patterns of yeast and Escherichia coli with reference to the abundance of isoaccepting transfer RNAs. J Mol Biol 1982;158(04):573-597

65 Ikemura T. Codon usage and tRNA content in unicellular and multicellular organisms. Mol Biol Evol 1985;2(01):13-34

66 Kanaya S, Yamada Y, Kudo Y, Ikemura T. Studies of codon usage and tRNA genes of 18 unicellular organisms and quantification of Bacillus subtilis tRNAs: gene expression level and species-specific diversity of codon usage based on multivariate analysis. Gene 1999;238(01):143-155

67 Yang J, Smith DK, Ni H, et al. SOX4-mediated repression of specific tRNAs inhibits proliferation of human glioblastoma cells. Proc Natl Acad Sci U S A 2020;117(11):5782-5790

68 Victor MP, Acharya D, Chakraborty S, Ghosh TC. The combined influence of codon composition and tRNA copy number regulates translational efficiency by influencing synonymous nucleotide substitution. Gene 2020;745:144640

69 Kanduc D. Changes of tRNA population during compensatory cell proliferation: differential expression of methionine-tRNA species. Arch Biochem Biophys 1997;342(01):1-5

70 Bayliss J, Karasoulos T, McLean CA. Immunosuppression increases JC polyomavirus large T antigen DNA load in the brains of patients without progressive multifocal leukoencephalopathy. J Infect Dis 2013;207(01):133-136

71 Maglennon GA, McIntosh PB, Doorbar J. Immunosuppression facilitates the reactivation of latent papillomavirus infections. J Virol 2014;88(01):710-716

72 García-Barchino MJ, Sarasquete ME, Panizo C, et al. Richter transformation driven by Epstein-Barr virus reactivation during therapy-related immunosuppression in chronic lymphocytic leukaemia. J Pathol 2018;245(01):61-73

73 Schoenfisch AL, Dollard SC, Amin M, et al. Cytomegalovirus (CMV) shedding is highly correlated with markers of immunosuppression in CMV-seropositive women. J Med Microbiol 2011;60(Pt 6):768-774

74 Koshy E, Mengting L, Kumar H, Jianbo W. Epidemiology, treatment and prevention of herpes zoster: a comprehensive review. Indian J Dermatol Venereol Leprol 2018;84(03):251-262

$75 \mathrm{Wu} \mathrm{Y,} \mathrm{Huang} \mathrm{H,} \mathrm{Luo} \mathrm{Y.} \mathrm{Management} \mathrm{of} \mathrm{hepatitis} \mathrm{B} \mathrm{virus} \mathrm{in}$ allogeneic hematopoietic stem cell transplantation. Front Immunol 2021;11:610500

76 Czech MM, Nayak AK, Subramanian K, et al. Reactivation of Chagas disease in a patient with an autoimmune rheumatic disease: case report and review of the literature. Open Forum Infect Dis 2021;8 (02):a642
77 Pegoraro F, Favre C. Post-transplantation lymphoproliferative disorder after haematopoietic stem cell transplantation. Ann Hematol 2021;100(04):865-878

78 Rosner BA, Cristofalo VJ. Hydrocortisone: a specific modulator of in vitro cell proliferation and aging. Mech Ageing Dev 1979;9(56):485-496

79 Phillips PD, Cristofalo VJ. Classification system based on the functional equivalency of mitogens that regulate WI-38 cell proliferation. Exp Cell Res 1988;175(02):396-403

80 Neuberger TJ, Kalimi O, Regelson W, Kalimi M, De Vries GH. Glucocorticoids enhance the potency of Schwann cell mitogens. J Neurosci Res 1994;38(03):300-313

81 Cai J, Zheng T, Lotz M, Zhang Y, Masood R, Gill P. Glucocorticoids induce Kaposi's sarcoma cell proliferation through the regulation of transforming growth factor-beta. Blood 1997;89(05): 1491-1500

82 Kawamura A, Tamaki N, Kokunai T. Effect of dexamethasone on cell proliferation of neuroepithelial tumor cell lines. Neurol Med Chir (Tokyo) 1998;38(10):633-638, discussion 638-640

83 Bourcier T, Forgez P, Borderie V, Scheer S, Rostène W, Laroche L. Regulation of human corneal epithelial cell proliferation and apoptosis by dexamethasone. Invest Ophthalmol Vis Sci 2000; 41(13):4133-4141

84 Atmani H, Chappard D, Basle MF. Proliferation and differentiation of osteoblasts and adipocytes in rat bone marrow stromal cell cultures: effects of dexamethasone and calcitriol. J Cell Biochem 2003;89(02):364-372

85 Rafacho A, Cestari TM, Taboga SR, Boschero AC, Bosqueiro JR. High doses of dexamethasone induce increased beta-cell proliferation in pancreatic rat islets. Am J Physiol Endocrinol Metab 2009;296 (04):E681-E689

86 Pickholtz E, Admon D, Izhar U, Berkman N, Levi-Schaffer F. Dexamethasone and salbutamol stimulate human lung fibroblast proliferation. World Allergy Organ J 2011;4(12):249-256

87 Zheng Y, Izumi K, Li Y, Ishiguro H, Miyamoto H. Contrary regulation of bladder cancer cell proliferation and invasion by dexamethasone-mediated glucocorticoid receptor signals. Mol Cancer Ther 2012;11(12):2621-2632

88 Gündisch S, Boeckeler E, Behrends U, Amtmann E, Ehrhardt H, Jeremias I. Glucocorticoids augment survival and proliferation of tumor cells. Anticancer Res 2012;32(10):4251-4261

89 Ninomiya E, Hattori T, Toyoda M, Umezawa A, Hamazaki T, Shintaku H. Glucocorticoids promote neural progenitor cell proliferation derived from human induced pluripotent stem cells. Springerplus 2014;3:527

90 Larson AA, Syverud BC, Florida SE, Rodriguez BL, Pantelic MN, Larkin LM. Effects of dexamethasone dose and timing on tissueengineered skeletal muscle units. Cells Tissues Organs 2018;205 (04):197-207

91 Grisé KN, Bautista NX, Jacques K, Coles BLK, van der Kooy D. Glucocorticoid agonists enhance retinal stem cell self-renewal and proliferation. Stem Cell Res Ther 2021;12(01):83

92 Natale C, Giannini T, Lucchese A, Kanduc D. Computer-assisted analysis of molecular mimicry between human papillomavirus 16 E7 oncoprotein and human protein sequences. Immunol Cell Biol 2000;78(06):580-585

93 Kanduc D, Stufano A, Lucchese G, Kusalik A. Massive peptide sharing between viral and human proteomes. Peptides 2008;29 (10):1755-1766

94 Trost B, Lucchese G, Stufano A, Bickis M, Kusalik A, Kanduc D. No human protein is exempt from bacterial motifs, not even one. Self Nonself 2010;1(04):328-334

95 Kanduc D, Shoenfeld Y. On the molecular determinants of the SARS-CoV-2 attack. Clin Immunol 2020;215:108426

96 Kanduc D. Severe acute respiratory syndrome coronavirus 2 (SARS-CoV-2): codon usage and replicative fitness. Glob Med Genet 2020;7(03):92-94 